\title{
ANÁLISE ETNOSSEMÂNTICA DE NOMES COMUNS DE ABELHAS E VESPAS (INSECTA, HYMENOPTERA) NA TERRA INDÍGENA PANKARARÉ, BAHIA, BRASIL
}

\author{
(Analysis of common names for bees and wasps [Insecta, \\ Hymenoptera] with cultural value for Pankararé Indians of \\ the village Brejo dos Burgos, Northeast Bahia, Brazil)
}

Eraldo Medeiros Costa Neto ${ }^{1}$

(Universidade Estadual de Feira de Santana - UEFS)

\begin{abstract}
This paper focuses on the ethnotaxonomy and significance of bees and wasps to the Pankararé Indians living in a semi-arid zone of the Northeast of the Bahia State, Brazil. The data were obtained by means of open-ended interviews performed with 14 individuals (nine men and five women) from Brejo do Burgo village. A total of 23 ethnospecies were recorded within the ethnocategory "abeia", the label used for both Apidae and Vespidae. These "abeias" are classified in two groups as "fierce bees" and "mild bees", and in three intermediate taxa depending upon whether or not they sting and, if so, if they can sting repeatedly. These insects play significant roles in the social, economical, and cultural life of this group.

Keywords: Ethnoentomology, ethnotaxonomy, traditional knowledge, semantics.

\section{RESUMO}

Este artigo focaliza a etnotaxonomia e importância de abelhas e vespas para os índios Pankararé, grupo residente em uma região semiárida do Nordeste

1. Mestre em Desenvolvimento e Meio Ambiente pela Universidade Federal de Alagoas (1998); Doutor em Ecologia e Recursos Naturais pela Universidade Federal de São Carlos (2003). Pós-doutorado em Biologia na UNAM (2005), onde trabalhou em etnoentomologia. Professor Titular da Universidade Estadual de Feira de Santana, onde atua como docente desde 1995 na disciplina Etnobiologia. Em Ecologia, atua nos seguintes temas: etnoentomologia, etnozoologia, conhecimentos tradicionais, zooterapia e medicina tradicional.
\end{abstract}


do Estado da Bahia, Brasil. Os dados foram obtidos mediante entrevistas semiestruturadas com 14 indivíduos (nove homens e cinco mulheres) da aldeia Brejo do Burgo. Um total de 23 etnoespécies foi registrado e "abeia" é o rótulo usado para apídeos e vespídeos. As "abeias" são classificadas em "abeias-brabas" e "abeias-mansas" e em três taxa intermediários dependendo da posse do ferrão e se conseguem ferroar repetidamente. Esses insetos desempenham significativos papéis na vida social, econômica e cultural desse grupo indígena.

Palavras-chave: Etnoentomologia, etnotaxonomia, conhecimento tradicional, semântica.

\section{Introdução}

Os seres humanos respondem às diversidades biológica, abiótica e sobrenatural que estão presentes nos ambientes onde vivem agrupando ou separando os elementos com base em similaridades e diferenças, levando em consideração não apenas as características intrínsecas (morfologia e anatomia) e extrínsecas (comportamento, ecologia etc.), como também as representações simbólicas no momento de reuni-los em categorias semanticamente definidas (Santos-Fita e Costa Neto, 2009). O universo léxico que compõe uma dada língua é o resultado de um longo processo biológico, histórico e cultural, que é traduzido em uma grande variedade de sons e símbolos usados para dar sentido a tudo o que existe ao redor das pessoas (Maffi, 1999). Cada idioma, em particular, apresenta uma visão única do mundo, uma vez que todas as percepções (ver, ouvir, tocar, cheirar e saborear) são canalizadas por hábitos linguísticos que favorecem alternativas de interpretação (Revel, 1994).

Estudos em etnotaxonomia biológica objetivam investigar como organismos vivos são percebidos, identificados, nomeados e classificados, buscando entender de que maneira os indivíduos categorizam (domínios etnossemânticos) e organizam (estruturas etnotaxonômicas) os elementos da natureza (Hunn, 1982, 1997; Hays, 1983; Brown, 1985; Berlin, 1992). No entanto, eleger os critérios de classificação denota certa subjetividade e viés por parte 
de quem a faz, pois tanto o significado emocional quanto as atitudes culturalmente aprendidas (representação afetiva) desempenham papéis importantes na formação e organização dos domínios etnozoológicos (Nolan e Robbins, 2001, Nolan et al., 2006). Tratar-se-ia, portanto, da correspondência entre o que é pensado (cognição), o que é dito (palavra) e o que é feito (ação) (Viertler, 2002). A classificação etnobiológica pode ser uma boa indicadora desse processo cognitivolinguístivo-comportamental (Couto, 2007).

$\mathrm{O}$ estudo da etnotaxonomia biológica tem importância tanto para compreender e entender a biodiversidade local quanto para analisar a universalidade da capacidade humana em classificar o mundo biológico. A estrutura cognitiva da diversidade biológica centra-se em "tipos naturais" (em um sentido amplo) de organismos que são linguisticamente reconhecidos e incluídos em um domínio semântico etnobiológico (Berlin, 1992). Considerando que os seres humanos, em várias partes do mundo, utilizam estratégias cognitivas semelhantes para classificar as coisas vivas e para organizar os conceitos biológicos, estudos em etnotaxonomia revelam, de fato, que o principal problema sempre recaiu em achar quais são as semelhanças ou diferenças que poderiam realmente ser importantes para fins de classificação. Diferentes autores defendem essa assertiva (Brown, 1984; Atran, 1990, 1998, Berlin, 1992, Ellen, 1993).

O presente artigo aborda os aspectos etnotaxonômicos de insetos sociais da ordem Hymenoptera (abelhas e vespas, com exceção de formigas) e sua importância para os índios Pankararé da região nordeste do Estado da Bahia, Brasil.

\section{Os Pankararé}

A Terra Indígena Pankararé ocupa uma área de 45.600 ha no nordeste do Estado da Bahia, localizada dentro da região conhecida como Raso da Catarina (Brazil, 1983), no quadrilátero formado pelas cidades de Paulo Afonso, Jeremoabo, Canudos e Macururé. A região é caracterizada pelo clima semiárido e vegetação típica de caatinga. Essa 
é a região mais seca da Bahia, com uma temperatura anual média de $27^{\circ} \mathrm{C}$ e precipitação de cerca de $400 \mathrm{~mm}$ ano (CEI, 1994).

Estudos sobre os Pankararé começaram nos anos 1970 e resultaram no reconhecimento do grupo como uma comunidade indígena. De acordo com Pinto (1991), os Pankararé parecem ser descendentes dos Gê; no entanto, estão altamente aculturados por meio da miscigenação com povos de diferentes grupos culturais e linguísticos. Eles são uma unidade linguística não diferenciada restrita à porção nordeste da Bahia.

Os sujeitos estão distribuídos em grupos agrícolas familiares na aldeia Brejo do Burgo. Os principais cultivos são milho, feijão e mandioca, plantados para consumo e para o comércio externo. A atividade agrícola é feita nos meses chuvosos (abril a agosto) devido às condições mais propícias do solo e disponibilidade de água. Os recursos proteicos são carne de bode, porco e frango, complementados com caça, mel e frutas nativas (umbu Spondias tuberosa, Anacardiaceae; murici Byrsonima gardneriana, Malpighiaceae; licuri Syagrus coronata, Arecaceae; castanha-de-caju Anacardium occidentale, Anacardiaceae) (Maia, 1992).

\section{Metodologia}

As observações aqui reportadas são baseadas em trabalho de campo realizado durante visitas mensais de cerca de três dias de julho a novembro de 1995 com moradores da aldeia de Brejo do Burgo. Foram entrevistados 14 indivíduos (nove homens e cinco mulheres) com idades entre 18 a 67 anos. Os homens se destacaram mais no conhecimento etnoentomológico das espécies de abelhas e vespas porque, provavelmente, são eles que caçam e coletam mel nativo. Apenas um especialista nativo na criação de abelhas Meliponinae foi entrevistado, sendo o principal consultor (Afonso Pankararé, 42 anos). Os entrevistados ganham a vida criando animais (bodes e porcos) e plantando milho, mandioca, feijão, melão e melancia, que são produtos comercializados na feira livre de Paulo Afonso. 
Por meio de uma abordagem êmica (Sturtevant, 1964; Posey, 1986), os dados foram obtidos a partir de entrevistas semiestruturadas, registrando-se o sistema de classificação etnoentomológico e os usos locais de abelhas e vespas pelos Pankararé. As entrevistas foram realizadas em português, pois os Pankararé são aculturados nesse idioma.

Os espécimes eram identificados pelos entrevistados antes e depois que eram coletados, com uso de rede entomológica, em seus ninhos ou quando visitavam as flores. Os insetos coletados eram mostrados aos indígenas para que falassem sobre seus nomes comuns, usos básicos, se criavam para coleta do mel, comportamento e presença em rituais. Os espécimes foram devidamente montados conforme os padrões usuais de coleções zoológicas e estão depositados no Museu de Zoologisa da Universidade Estadual de Feira de Santana. As espécies de vespas foram identificadas pelo Dr. Oton Marques, da Faculdade de Agronomia da Universidade Federal do Recôncavo Baiano; as espécies de abelhas foram identificadas pela Dra. Marina Siqueira, do Departmenta de Biologia da UEFS. Embora espécimes de manduri, mané-de-abreu, mandassaia e exuí não tenham sido coletados durante o trabalho de campo, seus nomes científicos são tentativamente fornecidos com base no catálogo de nomes comuns de insetos do Brasil (Lenko e Papavero, 1979; Buzzi, 1994). Os dados foram analisados segundo técnicas etnossemânticas (Conklin, 1962; Berlin, 1992; Couto, 2007) para entender o significado dos nomes de vespas e abelhas na cultura Pankararé.

\section{Resultados e Discussão}

Um total de 23 categorias de insetos é classificado como "abeias" pelos índios Pankararé. Neste lexema são incluídos aqueles himenópteros que produzem e estocam mel (abelhas e vespas eussociais, melíferas). O termo "abeia" aparece aqui entre aspas para contrastar com o vocábulo abelha, pois este último designa apenas insetos da superfamília Apoidea, excluindo as vespas. Na cosmologia Pankararé, 
todas as "abeias" são consideradas como seres vivos encantados e são protegidas da exploração humana por espíritos guardiães da natureza chamados encantados.

Bandeira (1993) provavelmente foi o primeiro pesquisador que registrou como os Pankararé percebiam e classificavam as "abeias". A precisão desses índios sobre as abelhas e vespas inclui detalhes morfológicos e adaptativos desses insetos ao ambiente semiárido. Por exemplo, o entrevistado especialista na criação de abelhas reconhece que esses insetos carregam o pólen (saburá) em suas pernas. Sabese que abelhas Apidae evoluíram a curbícula ou cesta de pólen, uma modificação estrutural derivada da tíbia traseira que é usada para o transporte de pólen, resina, cera e outras substâncias (Roubik, 1989). Os Pankararé conhecem, inclusive, as plantas nas quais as "abeias" retiram pólen observando a cor e o saber dos méis. Segundo um entrevistado:

“[...] aqui é o saburá (pólen). É dessa pequena massinha amarela que o mel é feito. Vem do sumo da flor [...]. A abeia não faz mel com outra coisa. Todas as abeias fazem mel do sumo [isto é, o pólen] da flor. O alimento dos fio é o saburá".

No ecossistema semiárido, as abelhas, em particular, destacamse dos demais insetos devido ao papel ecológico que desempenham como agentes polinizadores importantes das flores da caatinga (Machado, 1990).

Seguindo os princípios de categorização de Berlin (Berlin, 1992), o termo "abeia" representa o nível de classificação associado ao táxon forma de vida. Em contraste com o que foi observado na maioria dos sistemas de classificação etnobiológicos, o táxon mais inclusivo - nível de reino - é nomeado e rotulado como animali. Assim, as "abeias" e todas as demais etnocategorias faunísticas estão subordinadas a este táxon. O nível forma de vida "abeia", por sua vez, está dividido em duas categorias intermediárias. Se uma "abeia" mostra um comportamento defensivo agressivo, então é considerada como abeia braba. Essa categoria inclui a abelha Apis mellifera scutellata, sete espécies de vespas sociais da tribo Epiponini (espécies de ninhos 
de cartão), e uma espécie de Meliponinae localmente chamada arapuá (Trigona spinipes). Se não é agressiva, a etnoespécie é referida como abeia mansa. Todas essas "abeias" são meliponíneos (abelhas sem ferrão). Essa distinção implica no modo como os Pankararé lidam com as vespas e abelhas. Eles queimam folhas verdes perto do ninho ou da colmeia das espécies brabas para afastá-las ou mesmo matar os adultos; então eles coletam mel, cera e larvas.

Interessante observar que as "abeias" são classificadas em três táxons intermediários dependendo da presença ou ausência e da perda ou retenção do ferrão (Tabela 1). Os Pankararé colocam todas as vespas Epiponini na "linha" ou etnofamília daquelas "abeias" que retêm seu ferrão. Eles afirmam que alguns tipos de "abeias" brabas podem usar seus ferrões mais de 20 vezes. Essa etnofamília geralmente é designada como "linha do exu", onde exu é um táxon polissêmico que ocupa tanto essa posição intermediária quanto o nível genérico. A abelha-de-mel (Apis mellifera scutellata) compõe a "linha da oropa" porque essa "abeia" perde seu ferrão após uso. De acordo com um entrevistado:

Exu-verdadeiro, cangota, tarantantã, caraquile, exu-preto e exuí têm a mesma mordida, a mesma ferroada e a mesma linha. Elas podem morder mais de uma vez [...]. O ferrão de uma oropa é diferente, mais simples. Se uma morde você, ela deixa o ferrão em você e não pode mais morder porque não nasce outro ferrão".

O terceiro grupo intermediário é formado por 15 etnoespécies de "abeias" sem ferrão, a "linha da arapuá". As duas primeiras linhas incluem "abeias brabas"; a última é equivalente às "abeias" mansas. Embora a arapuá seja identificada como agressiva, falta-lhe o ferrão.

Zangões e operárias não são distinguidos, mas uma abeia mestra sim. Os consultores reconhecem que todas as "abeias" têm uma mestra vivendo no ninho, que é diferenciada das demais devido ao seu tamanho maior. A mestra é a rainha (Roubik, 1889).

O significado material e cultural dos recursos das "abeias", bem como o comportamento de ferroar de algumas espécies, podem explicar o reconhecimento fino dos genéricos dentro das etnofamílias. 
A classificação Pankararé de himenópteros sociais em "linhas" demonstra uma correspondência um-a-um às famílias científicas da taxonomia acadêmica (ver Tabela 1). Os táxons incluídos são diferenciados por critérios morfológicos e comportamentais, como forma e tamanho, padrão de cor, comportamento de nidificação, estrutura da colmeia, disposição do mel nos favos, produção de mel, agressividade etc.

Em contraste, os vespídeos sociais com ninhos abertos, as vespas dos gêneros Polistes e Apoica, são colocados à parte no genérico maribondo. Como os indígenas afirmam, "O maribondochapéu (Apoica pallens) e o maribondo-caboclo (Polistes canadensis canadensis) não são abeias porque não produzem mel”. No entanto, os Pankararé utilizam seus ninhos como recursos medicinais para tratar enfermidades localmente diagnosticadas, como tontura, asma e derrame. Outras espécies de Hymenoptera, como vespas-potes-debarro, abelhas mangangás, vespas e abelhas que nidificam no solo e espécimes solitários, são classificados como besouros, sendo que a maioria deles não possuem nomes locais. A etnotaxonomia desses insetos não foi completamente analisada e não será discutida conforma Tabela apresentada a seguir.

Tabela 1. Etnotaxonomia Pankararé de vespas e abelhas (Insecta: Hymenoptera) colocadas na forma de vida "abeia", mostrando as categorias intermediárias de etnofamílias de acordo com comportamento defensivo.

\begin{tabular}{|l|l|l|l|}
\hline Intermediário & Genéricos e específicos (espécies científicas) & Braba & Mansa \\
\hline Linha do exu (Vespidae, Polistinae, Epiponini) & $\mathrm{X}$ & \\
\hline & Cangota (Polybia occidentalis) & $\mathrm{X}$ & \\
\hline & Caraquile (Polybia paulista) & $\mathrm{X}$ & \\
\hline & Exu-de-cachorro (Protopolybia exigua exigua) & $\mathrm{X}$ & \\
\hline & Exu-preto (Polybia ignobilis) & $\mathrm{X}$ & \\
\hline & Exu-verdadeiro (Brachygastra lecheguana) & $\mathrm{X}$ & \\
\hline & Exuí (Polybia sp.) & $\mathrm{X}$ & \\
\hline
\end{tabular}


P. 237-251 Eraldo Medeiros Costa Neto

\begin{tabular}{|c|c|c|c|}
\hline \multicolumn{4}{|c|}{ Linha da oropa (Apidae, Apinae) } \\
\hline & Oropa (Apis mellifera scutellata) & $\mathrm{X}$ & \\
\hline \multicolumn{4}{|c|}{ Linha da arapuá (Apidae, Meliponinae) } \\
\hline & $\begin{array}{l}\text { Abeia-branca-do-fundinho-branco } \\
\text { (Frieseomellita silvestrii) }\end{array}$ & & $\mathrm{X}$ \\
\hline & $\begin{array}{l}\text { Abeia-branca-do-fundinho-vermeio } \\
\text { (Frieseomellita silvestrii) }\end{array}$ & & $\mathrm{X}$ \\
\hline & Arapuá-macho (Trigona spinipes) & $\mathrm{X}$ & \\
\hline & Arapuá-fêmea (Trigona spinipes) & $\mathrm{X}$ & \\
\hline & Cupira-boca-de-barro (Partamona cupira) & & $\mathrm{X}$ \\
\hline & Cupira-boca-de-berruga (Partamona cupira) & & $\mathrm{X}$ \\
\hline & Mandassaia (Melipona quadrifasciata?) & & $\mathrm{X}$ \\
\hline & Manduri (Melipona rufiventris?) & & $\mathrm{X}$ \\
\hline & Mané-de-abreu (Frieseomelitta varia ?) & & $\mathrm{X}$ \\
\hline & Mosquito-preto (Plebeia mosquito) & & $\mathrm{X}$ \\
\hline & Mosquito-remela (Friesella schrottkyi) & & $\mathrm{X}$ \\
\hline & Mosquito-verdadeiro (Tetragona angustula) & & $\mathrm{X}$ \\
\hline & Papa-terra (Cephalotrigona capitata?) & & $\mathrm{X}$ \\
\hline & Trombeta (Plebeia sp.) & & $\mathrm{X}$ \\
\hline & Uruçu (Melipona scutellaris) & & $\mathrm{X}$ \\
\hline
\end{tabular}

A construção de nomes específicos na sistemática etnobiológica é regularmente binominal, com o genérico modificado por um adjetivo que frequentemente designa algum caráter morfológico óbvio. Nomes específicos monominais também são encontrados nas etnotaxonomias, mas quando ocorrem eles são polissêmicos com respeito ao genérico superordenado. A distinção entre um táxon específico prototípico e polissêmico e seu genérico superordenado pode ser feita explicitamente pelo uso de um atributo marcante, o qual pode ser traduzido como "real", "original", "mais visível" ou "verdadeiro" nas formações linguísticas (Berlin, 1992: 90-96).

Dois exemplos de táxons específicos prototípicos apareceram na etnotaxonomia Pankararé. Exu-verdadeiro (Brachygastra lecheguana) é uma vespa social de corpo curto e coloração negra, com bandas 
transversais amarelas na ponta do abdome. Essa etnoespécie provavelmente é o protótipo devido à sua ferroada poderosa e quantidade de mel que ela estoca. Duas outras etnoespécies pertencentes ao genérico exu são: exu-de-cachorro (Protopolybia exigua exigua) e exupreto (Polybia ignobilis), conforme se pode identificar na Figura 1, apresentada adiante.

Seus ninhos são feitos de esterco de boi e considerados medicinais. Outro específico prototípico é exemplificado pelo mosquitoverdadeiro (Tetragona angustula) e, segundo Atenor Pankararé (55 anos), essa forma típica produz mais mel que as outras espécies de mosquito: mosquito-remela (Friesella schrottkyi) e mosquito-preto (Plebeia mosquito). Alguns informantes afirmaram que o mosquitoverdadeiro faz cerca de um litro de mel, enquanto mosquito-preto apenas produz metade. Seus méis têm valor medicinal.

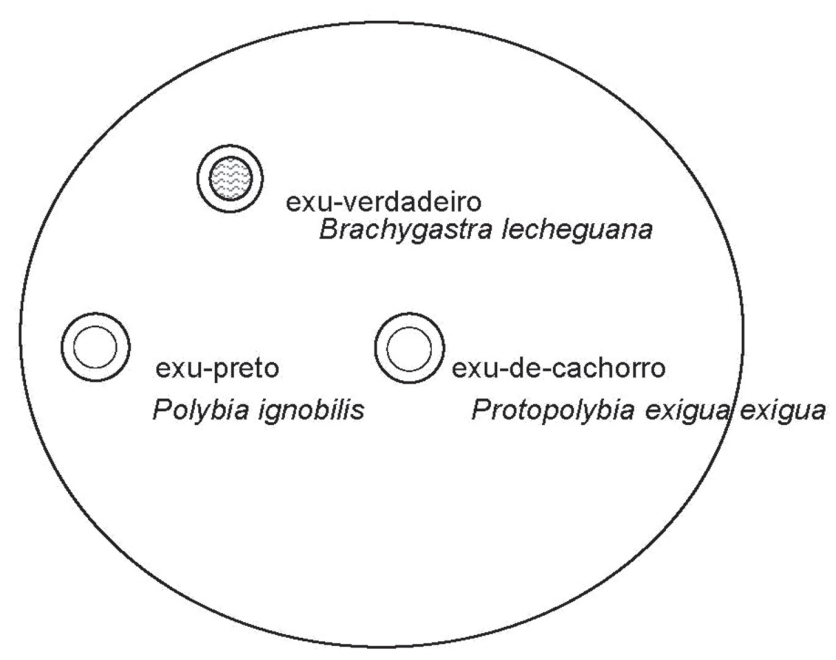

Figura 1: Representação diagramática de "abeias" do genérico exu.

O elemento prototípico está marcado com tracejados (segundo Berlin, 1992)².

2. Quero expressar minha gratidão ao Dr. Darrell Posey (já falecido) e Dr. Eugene Hunn pelos comentários ao manuscrito original; ao Dr. Oto Meira Marques e Dra. Marina Siqueira pela identificação dos espécimes. Sou muito grato aos Pankararé pela hospitalidade. 
Nomear por contraste binário é uma característica linguística comum em que um lexema primário é modificado para formar lexemas secundários, segundo uma dimensão semântica óbvia (Brown, 1979). Três conjuntos de táxons específicos são formados por esse processo no sistema de classificação entomológico Panakararé. Os táxons específicos incluídos no genérico abeia-branca são claramente marcados por contraste binário, no qual a coloração do abdome é a principal dimensão semântica. Enquanto a abeia-branca-do-fundinhobranco tem o abdome colorido de branco, o etnotáxon contrastante, abeia-branca-do-fundinho-vermelho, tem-no marcado de vermelho. A entrada da colmeia dos dois tipos é feita de cera. Taxonomicamente, os dois tipos de abeia-branca representam, provavelmente, variedades da mesma espécie Frieseomellita silvestrii.

O mesmo pode ser dito para cupira (Partamona cupira), apesar de nenhum espécime ter sido examinado pelo indígena especialista. A distinção entre cupira-boca-de-berruga e cupira-boca-de-barro alude ao material que é usado para construir a entrada da colmeia. A primeira reveste a entrada de seu ninho com cera, enquanto a segunda nidifica entro de um cupinzeiro e utiliza barro para construir a entrada da colmeia. O terceiro táxon genérico ilustrando contraste binário é a arapuá. Segundo os Pankararé, a dimensão semântica característica usada para separar os dois tipos de arapuá é a quantidade de mel produzida e o tamanho da entrada da colmeia. Diz-se que a abeia arapuá-macho produz mais mel que a arapuá-fêmea, e a entrada de sua comeia é mais comprida. Os Pankararé consideram seu mel saboroso. Essas duas etnoespécies correspondem a apenas uma espécie científica, Trigona spinipes. Aparentemente, a colmeia da arapuá-macho é mais velha e por isso a entrada é mais comprida que a da arapuá-fêmea. Os entrevistados disseram que a cupira lembra a arapuá, mas aquela é levemente maior e mais brilhante que a segunda.

As 23 categorias de "abeias" reconhecidas pelos Pankararé incluem genéricos monotípicos, genéricos polissêmicos, bem como nomes específicos binominais e monominais. O conhecimento de uma elaborada etnotaxonomia de insetos sociais indica a alta importância cultural de abelhas e vespas eussociais para os Pankararé. Os índios 
interagem com esses animais de diferentes maneiras, usando-os como alimento, preparando remédios tradicionais, consumindo mel em rituais religiosos e usando a cera para fazer ferramentas, como balas, ou para vedar panelas. Os Pankararé primariamente vão à caatinga buscar aquelas "abeias" cujo mel é de qualidade superior, como oropa e abelhas sem ferrão. Os informantes afirmam que a qualidade do mel depende da qualidade da árvore na qual a abelha construiu sua colmeia, da idade da colmeia e do tipo de florada. As vespas, por sua vez, produzem pouco mel e de baixa qualidade. Os Pankararé comercializam o mel na feira de Paulo Afonso, gerando renda para comprar produtos alimentícios, como açúcar, farinha e sal. Essa atividade é feita nos meses mais secos do ano quando a baixa produção agrícola coincide com uma maior produtividade de mel. Ainda, o mel é importante quando os homens vão caçar na caatinga, onde "água e alimento são disponíveis somente para aqueles que sabem como encontrar esses recursos" (Bandeira, 1993).

Não obstante a importância cultural e econômica das "abeias", os Pankararé apenas fazem a criação artesanal de uma espécie nativa: uruçu Melipona scutellaris. Essa "abeia" mansa é criada perto das casas em cavidades naturais ou ninhos artificiais chamados de cortiços, que são instalados sob os beirais das casas ou amarrados nos falhos de uma árvore próxima. A meliponicultura, no entanto, não tem sido uma prática comum e precisa ser mais bem entendida como uma estratégia de conservação potencial das espécies de abelhas da caatinga.

\section{Considerações finais}

Este trabalho registra os resultados do primeiro estudo entomológico Pankararé acerca da etnotaxonomia e importância cultural de vespas e abelhas sociais que produzem e estocam mel. Esses insetos são reconhecidos localmente como "abeias", grupo etnossemântico que é dividido em três táxons intermediários de acordo com a presença e ausência do ferrão e se podem ferrão repetidamente. Etnoespécies prototípicas e genéricos formados por contraste binário 
são reconhecidos, porém, mais estudos são necessários para esclarecer o tratamento etnotaxonômico de himenópteros além de Apidae e Vespidae Epiponini.

Recebido: 10/09/2012

Aceito: 22/11/2012

eraldont@hotmail.com

\section{Referências bibliográficas}

ATRAN, S. Folk biology and the anthropology of science: cognitive universals and cultural particulars. Behavioral and Brain Sciences, 21(4): 547-569, 1998.

Cognitive foundations of natural history. Londres: Cambridge University Press, 1990.

BANDEIRA, F. P. S. F. Etnobiologia Pankararé. Monografia. Salvador: Universidade Federal da Bahia, 1993.

BERLIN, B. Ethnobiological classification: principles of categorization of plants and animals in traditional societies. New Jersey: Princeton University Press, 1992.

BRASIL, Ministério das Minas e Energia. Projeto “RADAMBRASIL”. Aracaju/ Recife: geologia, geomorfologia, pedologia, vegetação e uso potencial da terra, Brasília: Ministério das Minas e Energia, 1983.

BROWN, C. H. Folk zoological life-forms: their universality and growth. American Anthropologist, 81(4): 791-812, 1979.

Language and living things: uniformities in folk classification and naming. New Brunswick: Rutgers University Press, 1984.

. Mode of subsistence and folk biological taxonomy. Current Anthropology, 26: 43-64, 1985.

BUZZI, Z. J. Coletânea de nomes populares de insetos do Brasil. Curitiba: Universidade Federal do Paraná, 1994.

Centro de Estatística e Informações. nformações básicas dos municípios baianos. Região Nordeste. Salvador: Governo do Estado da Bahia, 1994. 
COUTO, H. H. Ecolingüística: estudo das relações entre língua e meio ambiente. Brasília: Thesaurus, 2007.

Ellen, R. F. The cultural relations of classification: an analysis of Nuaulu animal categories from central Seram. Cambridge: Cambridge University Press, 1993.

HAYS, T. E. Ndumba folk biology and general principles of ethnobotanical classifications and nomenclature. American Anthropologist, 85: 592-611, 1983.

HUNN, E. The utilitarian factor in folk biological classification. American Anthropologist, 84: 830-847, 1982.

Tzeltal folk zoology: the classification of discontinuities in nature. Berkeley: University of California, 1997.

LENKO, K.; PAPAVERO, N. Insetos no folclore. São Paulo: Cons. Est. Art. Cienc. Hum, 1979.

MACHADO, I. C. S. Biologia floral de espécies de caatinga no município de Alagoinha (PE). Tese de Doutorado, USP, 1990.

MAFFI, L. Language and the environment. In: POSEY, D. A. (org.). Cultural and spiritual values of biodiversity, p. 22-29. Londres: ITP, 1999.

MAIA, S. M. Os Pankararé do Brejo do Burgo: campesinato e etnicidade. Monografia. Salvados: Universidade Federal da Bahia, 1992.

NOLAN, J. M.; ROBBINS, M. C. E. Emotional meaning and the cognitive organization of ethnozoological domains. Journal of Linguistic Anthropology, 11(2): 240-249, 2001.

NOLAN, J. M.; JONES, K. E.; MCDOUGAL, K. W.; MCFARLIN, M. J.; WARD, M. K. The lovable, the loathsome, and the liminal: emotionality in ethnozoological cognition. Journal of Ethnobiology, 26(1): 126-138, 2006.

PINTO, E. As máscaras-de-dansa dos Pancararu de Tacaratu (remanescentes indígenas dos sertões de Pernambuco). Nordeste Indígena, sér. Etnohistória, 2: 5-15, 1991.

POSEY, D. A. Topics and issues in ethnoentomology with some suggestions for the development of hypothesis-generation and testing in ethnobiology. Journal of Ethnobiology, 6(1): 99-120, 1986.

REVEL, N. Fleurs de paroles: histoire naturelle Palawan I: lens dons de Nägsalad. Paris: Editions Peeters, 1994. 
ROUBIK, D. W. Ecology and natural history of tropical bees. Cambridge: Cambridge University Press, 1989.

SANTOS-FITA, D.; Costa Neto, E. M. Sistemas de clasificación etnozoológicos. In: COSTA NETO, E. M.; SANTOS-FITA, D.; VARGASCLAVIJO, M. (orgs.). Manual de Etnozoología: una guía teórico-práctica para investigar la interconexión del ser humano con los animales, p. 67-94. Valencia: Tundra Ediciones, 2009.

STURTEVANT, W. C. Studies in ethnoscience. American Anthropologist, 66(30): 99-131, 1964.

VIERTLER, R. Métodos antropológicos como ferramenta para estudos em etnobiologia e etnoecologia. In: AMOROZO, M. C. M.; MING, L. C.; SILVA, S. M. P. (org.). Métodos de coleta e análise de dados em etnobiologia, etnoecologia e disciplinas correlatas, , pp. 11-29. Rio Claro: UNESP/CNPq, 2002. 